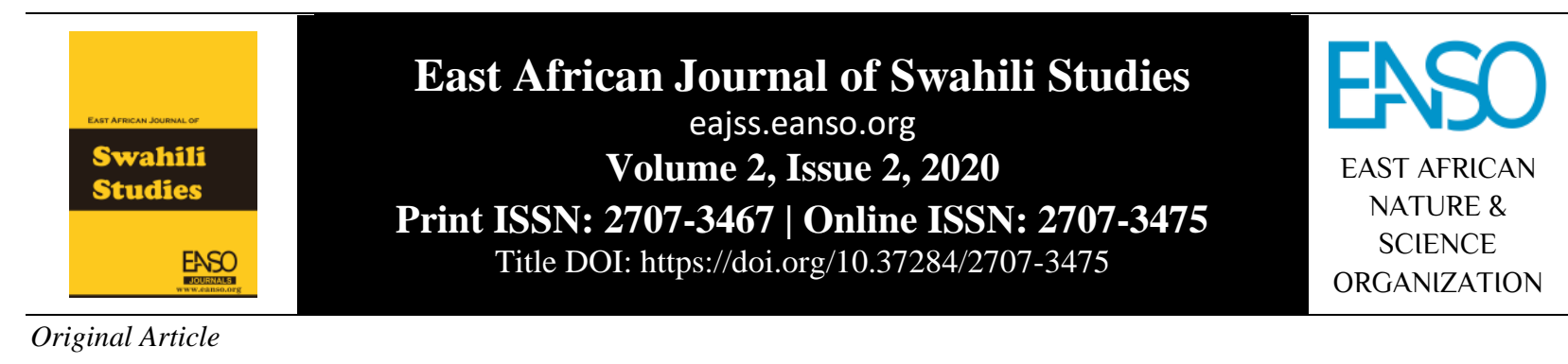

\title{
Vipengele vya Fasihi Simulizi Vinavyooana na Hali Katika Taaluma ya Sheria
}

\author{
Winnie Musailo Wekesa ${ }^{1}$, Dkt. Fred Wanjala Simiyu ${ }^{2}$ na Dkt. Nilson Isaac Opande ${ }^{3}$ \\ ${ }^{1}$ Chuo Kikuu Cha Masinde Muliro, S.L.P. 190 - 50100, Kakamega, Kenya. \\ ${ }^{2}$ Chuo Kikuu cha Kibabii, S.L.P. 1699 - 50200, Bungoma, Kenya. \\ ${ }^{3}$ Chuo Kikuu cha Kisii, S.L.P. 408 - 40200, Kisii, Kenya. \\ * Barua pepe ya mawasiliano: codle@mmust.ac.ke \\ *ORCID: https://orcid.org/0000-0001-8758-2936
}

DOI ya Nakala: https://doi.org/10.37284/eajss.2.2.193

\section{Tarehe ya Uchapishaji: IKISIRI}

13 Agosti 2020 Utafiti ulilenga kubainisha vipengele maalum vya Fasihi Simulizi vinavyooana na hali katika taaluma ya Sheria. Utafiti uliegemea uendeshaji wa kesi Istilahi Muhimu: kitamaduni miongoni mwa jamii ya Babukusu. Ili kuafikia lengo hili utafiti ulitumia mbinu ya kithamano ya utafiti. Idadi lengwa ya utafiti ilikuwa jumla Fasihi Simulizi,

Taaluma, Sheria. ya kesi za kitamaduni tulizohudhuria na kuzirekodi. Utafiti ulijumuisha michakato miwili ya uteuzi wa sampuli; ikiwa ni uteuzi sampuli kimaksudi na kinasibu. Uteuzi wa sampuli kimaksudi ulitumiwa kuchagua Chifu, naibu wa Chifu, mwenyekiti wa baraza la wazee na makala maalum yaliyoandikwa kuhusu Fasihi Simulizi na Sheria. Nao uteuzi wa sampuli kinasibu ulitumiwa kuteua mabaraza ya wazee na ya Chifu tulioyahudhuria. Waaidha, uchunguzi ulihusisha mbinu za uchambuzi wa yaliyomo, usaili na uchunzaji-shiriki. Njia hizi zilitumika kukusanya data nyanjani na maktabani. Matokeo ya utafiti yalichanganuliwa kithamano. Hii ni mbinu ya kuchanganua data ambayo mtafiti anaeleza na kufafanua matokeo ya utafiti wake kimaelezo. Kwa mujibu wa kesi tulizohudhuria, utafiti ulibaini kuwa kuna vipengele vya Fasihi Simulizi ambavyo vinaoana na hali ilivyo katika taaluma ya Sheria wakati wa uendeshaji kesi kitamaduni miongoni mwa jamii ya Babukusu. Kwa hivyo ni muhali kusema kuwa matini za Kifasihi Simulizi huwa zinachota, kunukuu, kugeuza, kuiga kwa namna ya kufumba, kubeza au kurejelea kwa njia moja ama nyingine matini za Kisheria. Hali kadhalika tanzu zenyewe za Fasihi Simulizi zinaingiliana na kuchangiana katika kutimiza mahitaji ya jamii kupitia shughuli mbalimbali. 


\section{APA CITATION}

Wekesa, W., Simiyu, F., \& Opande, N. (2020). Vipengele vya Fasihi Simulizi Vinavyooana na Hali Katika Taaluma ya Sheria. East African Journal of Swahili Studies, 2(2), 65-83. https://doi.org/10.37284/eajss.2.2.193

\section{CHICAGO CITATION}

Wekesa, Winnie, Fred Simiyu, and Nilson Opande. 2020. "Vipengele vya Fasihi Simulizi Vinavyooana na Hali Katika Taaluma ya Sheria”. East African Journal of Swahili Studies 2 (2), 65-83. https://doi.org/10.37284/eajss.2.2.193.

\section{HARVARD CITATION}

Wekesa, W., Simiyu, F. and Opande, N. (2020) "Vipengele vya Fasihi Simulizi Vinavyooana na Hali Katika Taaluma ya Sheria”, East African Journal of Swahili Studies, 2(2), pp. 65-83. doi: 10.37284/eajss.2.2.193.

\section{IEEE CITATION}

W. Wekesa, F. Simiyu, and N. Opande, "Vipengele vya Fasihi Simulizi Vinavyooana na Hali Katika Taaluma ya Sheria", EAJSS, vol. 2, no. 2, pp. 65-83, Aug. 2020.

\section{MLA CITATION}

Wekesa, Winnie, Fred Simiyu, and Nilson Opande. "Vipengele vya Fasihi Simulizi Vinavyooana na Hali Katika Taaluma ya Sheria". East African Journal of Swahili Studies, Vol. 2, no. 2, August 2020, pp. 65-83, doi:10.37284/eajss.2.2.193.

\section{UTANGULIZI}

Makala haya yaliazimia kuchunguza uhusiano wa kitaaluma uliopo baina ya Fasihi Simulizi na Sheria kwa kutathmini kesi zinapoendeshwa kitamaduni miongoni mwa Babukusu. Nyanja hii ina misingi yake katika mambo mawili makuu ambayo ni historia ya Sheria na Fasihi Simulizi. Masuala yanayochunguza uhusiano wa taaluma hizi yalijitokeza katika mikondo miwili; kwanza ni kuhusu dhana ya kuwepo kwa hali ya ukuaji wa kutoaminika juu ya iwapo sheria katika upekee wake ni chanzo cha thamani na maana ya mambo katika jamii. Ama iwapo ni lazima iingizwe katika muktadha wa utamaduni fulani, falsafa ama sayansi ya jamii ili kuwa na thamani au kutoa maana. Suala la pili lilihusu mtazamo wa kinyamavu unaokua kuhusu maana zinazojitokeza katika matini yoyote, iwe ya Fasihi Simulizi au ya Sheria na jinsi ya kuchanganua maana hizi. Maoni haya yalikuwa muhimu katika kukuza lengo la utafiti huu kwa sababu yalibainisha mitazamo miwili inayopambanua uhusiano wa Fasihi Simulizi na Sheria. Utafiti huu ulichanganua matini za Fasihi Simulizi na Sheria ili kudhihirisha uhusiano baina yake kijamii. Sheria katika Fasihi ni mtazamo unaojihusisha na namna ambavyo hali za kihistoria huwasilishwa kupitia Fasihi. Uhusiano baina ya taaluma hizi ni wa tangu miaka ya jadi kesi ziliposuluhishwa nje ya korti. Asili ya taaluma ya Sheria miongoni mwa Wagiriki kwa mfano ilihusishwa na usemi ulionuia kuwashawishi watu, kusema na kuandika, ikiwa na nia ya kuwasilisha majibizano kortini. Usemaji na uandishi zilikuwa ni mojawapo ya njia awali za diskosi na kuwasilisha malalamishi ya kesi. Sheria ilipokua zaidi kama taaluma, ilizuiliwa kuendelea kufanya kazi hii kwa njia hiyo. Hivyo basi, Sheria na Fasihi kila moja ikachukua mkondo wake. Fasihi ikawa inajihusisha sana na hisia za binadamu huku Sheria ikijihusisha na utunzaji wa usalama katika jamii. Maoni haya yanaonyesha asili ya taaluma ya Sheria kwa kuihusisha na Fasihi kwa jumla. Fasihi ya awali ya binadamu ilikuwa inahusu Usimulizi kwa sababu maandishi hayakuwepo. Hii ndiyo sababu utafiti huu uliturejesha kwenye Fasihi Simulizi huku ukiioanisha na taaluma ya Sheria. Akiandika mwaka 350 K.K, mwanafalsafa wa Ugiriki ya kale Aristotle alisema utawala wa Sheria ni bora kuliko mtu yeyote binafsi. Anazidi kusema kuwa mifumo ya Sheria huelezea haki na majukumu ya wananchi kwa njia mbalimbali. Kwamba taaluma ya Sheria ilikuwa chanzo kikuu cha uchunguzi wa kitaalamu wa baadhi ya nyanja zifuatazo zinazohusiana nayo: historia ya sheria, falsafa ya sheria, uchambuzi wa kiuchumi wa sheria au somo la kijamii kuhusu sheria. Fasihi Simulizi ni somo la kijamii hivyo basi linaweza kuingiliana na Sheria kitaaluma ili kuchunguza na kusuluhisha matatizo ya jamii kwa mtazamo jumuishi.

Mwingilianomatini katika kazi za Fasihi Simulizi na Sheria ni suala ambalo limekuwa likijitokeza sana katika shughuli anuwai za kijamii. Uhusiano huu ndio uliowafanya watafiti kuhusisha uchunguzi huu na korti ya Sirisia, ambayo awali ilionekana kujumuisha taaluma hizi mbili katika kusuluhisha kesi mbalimbali miongoni mwa Babukusu. Korti 
hii ilianza mnamo enzi za ukoloni nia kuu ikiwa ni kushughulikia washtakiwa ambao walikuwa wafuasi wa "Dini ya Msambwa". Wafuasi wa imani hii walichukuliwa kuwa waliwatatiza sana wakoloni wakati huo wakiongozwa na Elija Masinde wa Nameme. Korti hii ilianza kama sehemu ya korti ya Shieywe katika Kaunti ya Kakamega miaka ya 1920. Baadaye baina ya miaka ya 1948 - 1949 ililetwa katika tarafa ya Kabuchai ikiwa korti ya Kiafrika ya Tarafa ya Kabuchai. Hii iliambatana na hoja kuwa makao makuu ya wanadini ya Msambwa yalikuwa katika Mlima Elgon ambapo palikuwa na mapango matakatifu. Mapango haya ndiyo yalikuwa sehemu yao ya ibada kila walipotaka kusema na Mungu wao. Walienda hapo kuabudu na kutoa kafara kwa Mungu na miungu yao. Hivyo basi eneo la Sirisia likawa mwafaka kwa sababu mapango hayo yalikuwa katika sehemu ya chini ya Mlima Elgon inayopakana na Sirisia. Hivyo wakoloni walihamisha korti hii kutoka Kabuchai hadi Sirisia ili waweze kukabiliana na wafuasi hao kwa urahisi. Katika miaka hiyo ya enzi za kikoloni korti hii ilipoanzishwa hiyo ndiyo ilikuwa shughuli yake maalum.

\section{MBINU ZA UTAFITI}

\section{Muundo wa utafiti}

Kimsingi utafiti ulikuwa wa muundo wa kithamano na uliegemea mkabala wa kimfano. Mpangilio wa kimfano ni maarufu katika tafiti za sayansi ya jamii. Robson (2002) anasema kuwa mpangilio huu ni mchakato wa kufanya utafiti ambao huhusisha chunguzi za kihunzi fulani maalum katika hali yake halisi kimatini kwa kutumia vianzo kadhaa vya ushahidi. Utafiti unaohusisha mpangilio huu kwa kiasi fulani huruhusu matumizi ya elektroniki ambayo huhusisha kuuliza watafitiwa maswali, kutazama yanayoendelea na kurekodi kisha kuchanganua (Bassey, 1999).

Muundo wa kithamano uliwaongoza watafiti kukusanya habari kwa kuwahoji wasailiwa ambapo katika utafiti huu waliohojiwa ni mwenyekiti wa baraza la wazee ya jamii ya Babukusu, Chifu wa kata ya Sirisia na naibu Chifu wa kata ndogo ya Namono. Chifu na naibu wake waliteuliwa kwa sababu maeneo wanayosimamia ndiyo yalikuwa makao ya korti maarufu iliyoendesha kesi kitamaduni miongoni mwa Babukusu. Sababu nyingine ilitokana na tajriba yake ya kushikilia wadhifa huo kwa zaidi ya miaka ishirini na tano. Data inayokusanywa kwa muundo huu hutokana na kuuliza maswali na kujaza kwenye dodoso zilizoandaliwa awali. Maoni ya watafitiwa hutumiwa kutoa maamuzi kuhusu suala linalotafitiwa. Oso na Onen (2005) wanasema kwamba muundo huu wa utafiti ni bora zaidi kwa sababu husaidia pakubwa kukusanya data inayoonyesha ukweli na uhalisia wa suala la utafiti. Aidha muundo huu hufuata utaratibu maalum hivyo kutoa ujumbe wa kuaminika. Muundo huu uliwasaidia watafiti kutathmini, kubainisha na kuonyesha matumizi ya vipengele vya Fasihi Simulizi katika taaluma ya Sheria.

\section{Mahali pa utafiti}

Utafiti huu ulifanywa katika sehemu mbili ambazo ni maktabani na nyanjani. Tulijifungia katika maktaba kwa sababu baadhi ya data zilihitaji kudurusu vitabu, majarida na majuzuu kuhusu taaluma ya Sheria, Semantiki, nadharia ya utafiti na vipengele vya Fasihi Simulizi. Kazi za maktabani zilikuwa mwongozo bora kuhusu mada yetu. Sababu ya kuchagua maktaba ni kwamba utafiti huu ulilenga kupata na kusoma maandiko mbalimbali yanayohusiana na utafiti, hivyo katika maktaba ndiko kulikopatikana machapisho mbalimbali ambayo yalitupatia data za msingi na data za upili za utafiti wetu. Kando na kuhakiki machapisho kutoka katika maktaba hizo, watafiti walipitia pia tovuti mbalimbali zenye maandiko yanayohusiana na utafiti. Aidha tulienda nyanjani ili kujumuisha maoni ya washika dau mbalimbali katika utafiti huu. Tulizuru Kaunti ya Bungoma; Kaunti ndogo ya Sirisia na kuhudhuria mabaraza ya wazee na ya Chifu ili kushiriki na kushuhudia namna kesi zinavyoendeshwa kitamaduni. Vilevile tulitembelea makavazi ya Kakamega kutafuta data za uendeshaji wa kesi za kitamaduni zilizohifadhiwa huko. Data hizo zilihusu kesi za jamii ya Babukusu iliyokuwa inatafitiwa ambazo zimehifadhiwa kutokana na faili za korti ya Sirisia ilipokuwa mahakama ya kitamaduni. Kwa hivyo chaguo la makavazi haya kama mahali pa utafiti huu lilitokana na upatikanaji wa nyaraka mbalimbali za kesi za kitamaduni na data za nyanjani zilizowezesha watafiti kufikia malengo ya utafiti wao. 
Kaunti ya Bungoma ni mojawapo ya Kaunti arobaini na saba nchini Kenya. Vilevile ni kati ya Kaunti nne za eneo la Magharibi ya Kenya. Inapakana na Kaunti ya Kakamega upande wa Mashariki, Kaunti ya Busia upande wa Magharibi na Trans Nzoia upande wa Kaskazini. Idadi ya watu katika Kaunti hii ni 1,375,063 na eneo zima ni kilomita 2,069 (chanzo ni Wizara ya Mipango, Bungoma).

\section{Usampulishaji}

Kwa vile idadi ya umma lengwa ilikuwa kubwa watafiti walichagua sampuli ndogo ili kuiwakilisha. Kutokana na sampuli hii matokeo yaliweza kujumlishwa kwa watafitiwa wote. Kulingana na Gay (2002) sampuli na usampulishaji ni muhimu kwa sababu huwezesha kubaini kiwakilishi cha sampuli kwa matumizi ya jumla. Ni kuteua sampuli kutoka kundi lengwa ili itumike katika utafiti kama kiwakilishi cha kundi lolote linalotafitiwa kwa kuwa sio rahisi kutafiti kundi lengwa zima. Zipo mbinu mbalimbali ambazo hutumiwa katika uteuzi wa sampuli. Sampuli ya utafiti huu ilitumia mbinu mbili ambazo ni mbinu ya uteuzi sampuli kimaksudi na mbinu ya uteuzi nasibu.

\section{Umma lengwa}

Utafiti ulilenga jamii ya Babukusu ambao idadi yao kubwa inaishi katika Kaunti ya Bungoma eneo la Magharibi mwa Kenya na Kaunti ya Trans Nzoia. Hata hivyo kuna wanajamii hii wengine wanaoishi katika sehemu mbalimbali za nchi ya Kenya. Vilevile utafiti huu ulichunguza vipengele bainifu vya utamaduni wa jamii hii ambavyo ndivyo Fasihi Simulizi yake. Vipengele hivi vya Fasihi Simulizi viliweza kufungamanishwa na jinsi vinahusiana na uendeshaji wa kesi za kitamaduni miongoni mwa Babukusu. Watafiti pia walizuru makavazi ya korti pale Kakamega kwa sababu huko ndiko kulikohifadhiwa rekodi na faili za kesi zilizofanyika Sirisia ilipokuwa korti ya kitamaduni. Faili hizo zilihifadhiwa Kakamega huko ndiko kuliko makao makuu ya makavazi eneo la Magharibi.

\section{Sampuli}

Kwa mujibu wa Spata (2003) na Kombo na Tromp (2006), sampuli ni jumla ya wanachama wote, mtu mmoja, kikundi au elementi wakiwemo wanyama, vitu na matukio ambayo mtafiti amekusudia kuyahusisha katika kukusanya data za utafiti wake. Utafiti ulijumuisha michakato miwili ya uteuzi wa sampuli; ya kimaksudi na kinasibu. Uteuzi maksudi ulitumiwa kuchagua makala maalum yaliyoandikwa kuhusu tulichotafitia na makavazi ya korti. Vigeu hivi viwili viliteuliwa kwa kuzingatia sifa kuu za sampuli tuliyohitaji. Nao uteuzi wa sampuli kinasibu ulitumiwa kuteua mabaraza ya wazee na Chifu yaliyohudhuriwa. Mbinu hii ilinuia kuwapatia watafitiwa wote nafasi sawa ya kuweza kuteuliwa na kushiriki katika utafiti. Ili kupata data tulitumia hifadhi ya matukio ya data za uendeshaji wa kesi za kitamaduni.

\section{Mbinu ya uteuzi wa sampuli kimaksudi}

Uteuzi wa sampuli kimaksudi ni kuchagua sampuli kwa kuzingatia sababu mahususi. Katika mbinu hii uteuzi hulenga madhumuni maalum, sampuli huteuliwa kwa kuzingatia vigezo maalum ambavyo vitakuwa na uhusiano na maswali ya utafiti ili yaweze kujibiwa kwa urahisi na ukamilifu (Powell na Conway, 2004). Ni mbinu iliyotumiwa kuchagua watafitiwa walio na sifa na ujumbe muhimu unaoafiki malengo ya utafiti. Hii ilipunguza kutumia muda mrefu kwa utafiti.

Kombo na Tromp (2006), wanasema, ni uteuzi ambao mtafiti kwa makusudi hulenga kuteua kundi la watu ambao anaamini kuwa ni raslimali anayoitegemea katika utafiti wake. Mbinu hii ilitumiwa kuteua Kaunti ndogo ya Sirisia, makavazi ya nyaraka za korti ya Kakamega na kiongozi wa baraza la wazee la Babukusu. Sababu za kuteua Kaunti ndogo ya Sirisia ni kwa vile huko ndiko kulikokuwa na korti ya Kiafrika iliyoendesha kesi za Babukusu kwa misingi ya utamaduni wao. Aidha sehemu hii ya Sirisia ilitoa mandhari mwafaka ya mashinani ambako machifu waliendesha mikutano na mabaraza kwa lugha asilia ya Kibukusu. Kwa upande mwingine makavazi ya korti ya Kakamega yaliteuliwa kwa sababu huko ndiko kumehifadhiwa faili za kesi za zilizoendeshwa kitamaduni kutoka korti ya Sirisia. Naye kiongozi wa baraza la wazee ililazimu kuteuliwa kwa sababu yeye huwa ni mmoja pekee kwa kila muhula wa uongozi, vilevile palikuwa na haja ya kumsaili mtendaji wa mivigha ya khuswala kumuse/lufu. 


\section{Mbinu ya Uteuzi nasibu}

Mbinu hii huwawezesha watafiti kuteua sampuli bila ubaguzi. Kila mtafitiwa lengwa ana nafasi sawa ya kushiriki katika sampuli ya utafiti. Kothari (2013) anaelezea mbinu ya uteuzi nasibu kuwa, ni ile inayotumika kuteua sampuli ya wahojiwa miongoni mwa kundi kubwa la wahojiwa wenye sifa sawa bila kubagua. Sampuli katika mbinu hii, iliteuliwa kuwakilisha kundi kubwa bila kujali sifa ya mteuliwa kwani kila mmoja katika kundi hilo huwa na sifa sawa na mwingine. Kutokana na walengwa wote kuwa na sifa sawa au zinazofanana, watafiti waliamua kuteua sampuli nasibu ya mabaraza ya chifu na ya wazee. Katika mabaraza haya wanaohudhuria ni watu wa jinsia ya kiume na ya kike mradi amehusika katika kesi inayoendeshwa. Hii iliwapa watafitiwa wote nafasi sawa ya kushiriki katika utafiti. Uteuzi wa kundi hili ulizingatia jinsia kwa sababu utafiti huu uliamini kuwa watu wa jinsia tofauti wanaweza kutoa maoni na mtazamo tofauti hususan pale palipokuwa na data za ujinsia. Sampuli hii ilisaidia kubainisha mikakati ya mwingilianomatini wa vipengele vya Fasihi Simulizi ya Babukusu na taaluma ya Sheria na kuonyesha kufanana na kutofautiana kuliopo katika taaluma hizo.

\section{Mbinu za kukusanya data}

Wataalamu mbalimbali akiwemo Cohen (2000) wanathibitisha kuwa, katika ukusanyaji wa data za utafiti hakuna mbinu moja inayojitosheleza na hivyo kupendekeza matumizi ya mbinu zaidi ya moja ili kupata taarifa sahihi katika sayansi ya jamii. Kwa mantiki hii, utafiti huu umetumia mbinu kuu tatu za ukusanyaji data ambazo ni usaili, uchambuzi wa yaliyomo na uchunzaji-shiriki. Tulienda maktabani ili kusoma vitabu, majarida, majuzuu na magazeti kuhusu dhana za Fasihi Simulizi, Sheria na Semantiki. Vilevile tulienda nyanjani ili kupata rekodi na faili za kesi zilizohifadhiwa katika makavazi ya korti kule Kakamega. Mbinu ya uchunzaji-shiriki ilitumiwa kwa watafiti kuhudhuria mabaraza ya wazee na Chifu wakati wa uendeshwaji wa kesi mbalimbali. Hii ni kwa sababu ya kutazama na kurekodi namna kesi zinavyoendeshwa na kuendesha mahojiano ya moja kwa moja kwa watafitiwa hawa. Maswali tofauti yaliandaliwa kwenye dodoso mbalimbali kwa kila kikundi. Aidha tuliweza kishiriki katika uchunzaji kwenye vikao teule vya mabaraza ili kutambua na kubainisha vipengele mahususi vya Fasihi Simulizi ya Babukusu vinavyotumiwa katika uendeshaji wa kesi. Miongoni mwa vipengele vilivyotafitiwa ni mafumbo, methali, semi, vitendawili na hadithi fupi: ili kuonyesha namna ufumbaji uliomo unavyotumika kuwasilisha ujumbe na maana kwa wahusika kisha kufikia muafaka bila utata.

\section{Mbinu ya uchambuzi wa yaliyomo}

Jilala (2014) anasema mbinu hii huhusu uchambuzi wa jumbe zilizomo katika nyaraka kama vile vitabu, magazeti na maudhui ya maneno ambayo yanaweza kuwa ya maandishi au mazungumzo. Uchambuzi wa yaliyomo katika utafiti huu uliwezeshwa kwa kuelekezwa na maswali tuliyoandaa kwenye mwongozo wa uchambuzi wa yaliyomo.

\section{Mbinu ya usaili}

Haya ni majibizano ya ana kwa ana kati ya watu wawili au zaidi kwa lengo la kukusanya data au maoni kuhusu suala la kiutafiti (Kothari 2004). Katika utafiti huu watafitiwa waliulizwa maswali ya ana kwa ana ambayo mtafiti aliyaandaa awali ili kuhakikisha kuwa mtu anatoa hoja na maoni yaliyo ndani ya malengo ya utafiti. Aidha ni mbinu ya kukusanyia data ambayo inahusu mahojiano ya ana kwa ana kati ya mtafiti na mtafitiwa (Kothari, 2013). Ni mbinu inayowawezesha watafiti kupata maoni kamili na hisia za mtafitiwa. Mtafiti ana uhuru wa kufafanua zaidi maswali na kumweleza mtafitiwa ili apate ujumbe mwafaka unaohusu malengo yake ya utafiti. Usaili unaweza kuwa wa uso kwa uso, kupitia kwa simu au wavuti. Kupitia mbinu hii watafiti waliwahoji Chifu wa kata ya Sirisia, naibu wa Chifu kata ndogo ya Namono na mwenyekiti wa baraza la wazee la Babukusu ili kuweza kupata data zaidi zilizowezesha maswali ya utafiti kujibiwa na hivyo kukamilisha malengo ya utafiti huu.

\section{Mbinu ya uchunzaji-shiriki}

Mbinu ya uchunzaji inaweza ikatekelezwa kupitia kushiriki kwa watafiti katika tukio husika, huku wakitazama au watafiti kutazama huku wamejitenga na wasailiwa. Katika utafiti huu, mbinu ya uchunzaji kwa watafiti huku wamejitenga 
na wasailiwa ilitumiwa kwa lengo la kukusanya data isiyoathiriwa na mtazamo wa watafitiwa. Mbinu hii ilihusisha walengwa wote walioshiriki katika mabaraza ya uendeshaji kesi ambazo zilihudhuriwa na watafiti. Watafiti walikusanya nyimbo, ngano, methali, vitendawili, misemo na mashairi, kwa hisani ya mbinu hii. Aidha, kupitia mbinu hii, watafiti waliweza kunasa (kutumia kinasa sauti, kamera na video kamera) miondoko, miktadha ya utendaji, maleba na washirika maalum kwa kila utanzu uliokusanywa. Haya yalisaidia mno katika shughuli ya kufasili na kuchanganua data husika. Kothari (2004) anatetea matumizi ya mbinu ya utazamaji kwa kusema:

Ni data ambayo hukusanywa kutokana na ushahidi thabiti wa mambo yanayotokea wakati uo huo; sio kuhusu yaliyopita wala yatakayokuja. Mbinu hii pia, humsaidia mtafiti kukwepa vizingiti kama vile kukumbana na wasailiwa ambao wanadanganya au wanakataa kutoa taarifa muhimu, kwani mtafiti hujionea yeye mwenyewe.

Katika muktadha wa utafiti wetu mbinu hii ilijumuisha njia za kushuhudia na kushiriki katika kukusanya data. Mbinu ya kushiriki iliwawezesha watafiti kujumuika pamoja na wasailiwa katika utendaji wa uendeshaji kesi. Kwa kufanya hivyo, walipata fursa ya kuhisi kama walivyohisi watafitiwa, na kukusanya data faafu kuhusiana na utafiti huu.

\section{Uwasilishaji na uchanganuzi wa data}

Data iliwasilishwa na kuchanganuliwa kwa njia ya kithamano. Kulingana na Enon (1998), mkabala wa kithamano ni njia ya kuchanganua data ambayo mtafiti hueleza na kufafanua matokeo ya utafiti wake kwa kutumia maelezo. Naye Kress (2008), anasema mkabala huu hueleza vipi na kwa nini matukio yanatokea. Utaratibu wa uchanganuzi data kithamano hufuata hatua sita kama wanavyodai (Creswell, 2003; Jwan \& Ong'ondo, 2011). Hatua ya kwanza ilikuwa kunakili matokeo ya usaili vile yalivyotolewa na kuandikwa kwenye dodoso na kwa mwongozo wa uchambuzi wa yaliyomo kama ilivyohusiana na utafiti huu. Hatua ya pili ilikuwa kurekodi kwa kunasa sauti kutokana na matukio ya uchunzaji-shiriki. Kwenye hatua nyingine sauti zilizonaswa wakati wa mahojiano na kushiriki zilifafanuliwa kwa kuandikwa. Baadaye zilitumika kama data msingi katika uchanganuzi na uwasilishaji wa matokeo.

Kwa hivyo mkabala huu ulikuwa mwafaka kwa utafiti huu kwa vile kulikuwa na data zilizotokana na majibu ya kimaelezo yaliyogusa maoni ya watafitiwa. Data iliyokusanywa ilipangwa kwa utaratibu ili kuwezesha uchanganuzi kufanywa. Nadharia iliyoongoza uchunguzi huu ya Mwingilianomatini ilitumika kudhihirisha uhusiano wa kimatini za Sheria na Fasihi Simulizi kwa kuhakiki na kuchanganua matini hizi. Waaidha, utafiti ulionyesha namna vipengele bainifu vya Fasihi Simulizi hufumbwa ili kuleta utata wakati kesi zinapoendeshwa kitamaduni. Mwingliano huu ulitathminiwa zaidi kwa kuhusisha matumizi ya Semantiki ili kufafanua maana zilizochanuzwa kutokana na ufumbaji katika lugha teule ya kifasihi iliyotumika.

\section{Uhalali na uthabiti wa vifaa}

Vifaa vilivyotumika katika utafiti huu ni dodoso, ratiba ya uchunzaji na mwongozo wa uchambuzi wa yaliyomo. Dodoso ilitumiwa kupata data kutoka kwa kiongozi wa wazee wa jamii ya Babukusu, Chifu na naibu wa Chifu. Hii ilisaidia watafiti kudadisi kwa undani watafitiwa ili kupata habari za kina kuhusu uendeshaji wa kesi za kitamaduni miongoni mwa Babukusu kwa kushirikisha Fasihi Simulizi na Sheria. Ratiba ya uchunzaji ilitumiwa kunakili matukio ya moja kwa moja katika sherehe na utendaji wa mivigha ya Fasihi Simulizi ya Babukusu. Mivigha maalum iliyoshuhudiwa ilikuwa lufu na sisinini. Kwa upande mwingine mwongozo wa uchambuzi wa yaliyomo ulitupa data pana na ambayo ilishirikisha mambo anuwai kutoka kwa vitafitiwa. Maswali yaliyoandaliwa kwenye kifaa hiki yalielekeza utafiti kushughulikia vipengele faafu vinavyohusina na malengo ya utafiti pekee. Maswali yalihitaji majibu yanayojumuisha maelezo, ufafanuzi, kutathmini na usanisi. Kifaa hiki kilitumika kuelekeza uhakiki na usomaji wa makala na maandishi mbalimbali kuhusu mada inayochunguzwa.

\section{Maadili ya kitafiti}

Maadili huhusu mienendo binafsi ya mtafiti na huwa kama kielelezo cha tabia za mtu. Leedy (1997) anasema utafiti haupaswi kufanywa kabla ya 
malengo na manufaa yake kuwekwa bayana kwa watafitiwa. Ni hatia kumhadaa msailiwa yeyote akubali kushiriki katika utafiti wowote ule, bila ya yeye kujua manufaa na au hasara zinazotokana na kushiriki au kutoshiriki kwake katika kutoa taarifa zinazohusiana na utafiti. Kwa hivyo watafiti waliwaelezea watafitiwa malengo na umuhimu wa kufanya utafiti huu. Kisha watafiti walihakikishia watafitiwa uhifadhi na usiri wa yote watakayoyasema. Pale ambapo mtafitiwa hakutaka kutajwa au maelezo yanayoelekea kumtambua yasitolewe, wachunguzi walimhakikishia hilo. Tulitafuta ridhaa ya watafitiwa kabla ya kunasa sauti au matendo yao kwa namna yoyote ile. Pia tulitambua kazi za wataalam wengine kwa kuwanukuu na kuwajumuisha katika marejeleo ili kuepuka wizi wa kitaaluma.

\section{MATOKEO YA UTAFITI}

Uchunguzi ulibaini kuwa kuna vipengele vya Fasihi Simulizi vinavyodhihirika katika taaluma ya Sheria. Hali hii inathibitika kupitia maoni ya Msokile (1992) ambaye anasema Fasihi Simulizi ndiyo aina ya pekee ya sanaa inayotumia lugha kujitambulisha au kujitofautisha na aina nyigine za sanaa. Zaidi sana ni kuwa Fasihi Simulizi ni mfumo unaoweza kuainishwa na kuchanganuliwa katika tanzu mbali mbali zinazojitegemea na kujikamilisha kiutendakazi kwa kuingiliana na taaluma nyinginezo. Isitoshe matokeo haya yanakamilika zaidi kupitia nadharia ya utafiti kwa misingi ya mhimili wa kwanza ambao unasema kwamba, hakuna matini yoyote inayoweza kuangaliwa peke yake, kwa upekee wake na kwa kujitegemea, bila kuhusishwa na matini nyingine iliyotangulia ambayo kwa njia moja au nyingine inahusiana na matini hiyo mpya. Matini hutegemeana. Hii ina maana kuwa, kuna uhusiano na utegemezi kwa kazi za fasihi na taaluma nyinginezo, na kwa sababu hiyo, si rahisi kuzungumzia uasili au upekee wa kazi fulani, kwani kwa vyovyote vile itakuwa imetokana na kazi zingine zilizotangulia au zilizopo.

Kazi za kifasihi zinaundwa kutokana na mifumo ya kanuni na tamaduni mbalimbali zilizowekwa na kazi zingine tangulizi za kifasihi ambapo taswira zinazohusu maisha ya kawaida na uhusiano wa kimaana huunganishwa na kubadilishwa. Kutokana na vikao vya mabaraza ya Chifu tulivyohudhuria matumizi ya lugha yalionyesha utukiaji wa vipengele kadha vya Fasihi Simulizi katika uendeshaji wa kesi mbalimbali. Kwa mfano katika kikao kimoja cha tarehe 22/02/2019, katika kijiji cha Khamoto kesi iliyohusu mvulana aliyempachika msichana uja uzito kilichobainika ni kuwa wazee wa ukoo ndio waliohusishwa pakubwa katika utatuzi wa kesi za aina hii. Utaratibu mwingine ni kwamba kando na msichana, watu wa jinsia ya kike hawa kuhusishwa pakubwa katika uendeshwaji wa kesi hizi isipokuwa katika muktadha na hitaji maalum. Kutokana na kesi hiyo misemo kadha ya Kibukusu ilitumika mfano:

\section{Sibele sia embusi (Kuwa na uja uzito).}

Katika msemo huu; sibele ni titi la mnyama, sia ni la na embusi ni mbuzi. Maana ya juu ya msemo ni titi la mbuzi lakini maana yake ya ndani ni kuwa na uja uzito. Pia kulikuwa na matumizi ya msamiati sisombo/kamafuki/khuesia ni istilahi ambazo zina maana zifuatazo; sisombo ni uja uzito, kamafuki ni damu na khuesia ni kupata mimba. Zilitumika ili kuficha makali ya maana ya uja uzito. Hali kadhalika kulikuwa na matumizi ya methali ya kwanza ikiwa ni:

\section{Mkenda mbola kola ebunyolo (Mtu} anayetembea polepole hufika mbali).

Kwenye Methali hii; mkenda ni mtu anayetembea, mbola ni kwa mwendo wa polepole, kola ni kufika au kuwasili na Banyolo ni msemo unaotumiwa na jamii ya Babukusu kurejelea Wajaluo ambao walionekana kuwa mahali wanakoishi ni mbali. Kwa hivyo maana ya methali hii ni kwamba mtu anayeenda kwa mwendo wa polepole anaweza kufika mbali. Methali hii ilitumiwa kumtahadharisha mhusika Situma kwamba asiwe haraka kukana kumtunga msichana yule mimba kisha mambo yatokee kinyume baada ya kujifungua. Huenda mtoto atakayezaliwa aje kuwa wa manufaa siku za baadaye. Licha ya mvulana kukana ule uja uzito, baada ya msichana kujifungua kina mama wa ukoo wa mvulana hutumwa na bidhaa ili kuenda kuchunguza iwapo mtoto aliyezaliwa ni damu yao. Zaidi ya hayo methali nyingine ilikuwa ni:

3. Kumusuni kuli khu mutayi (Bahati huja mara moja). 
Kumusuni ni kipande cha mti kifupi ambacho hutumika kama silaha na mara nyingi kilitumiwa ili kujikinga. Kuli ni kuwa ilhali khu ni kwa mutayi ni yule wa kwanza. Hivyo methali hii ina maana kuwa kile ambacho umetangulia kukipata huenda ndiyo riziki yako ya pekee uliojaliwa na Mungu. Hiyo ndiyo silaha ya kujikinga na hali yoyote ya hatari ambayo inaweza kutokea. Hivyo ukiitupilia mbali kisha baadaye ukaja kukosa kuambulia bahati sawa na hivyo utakuja kujuta. Methali hii vilevile ilimrejelea Situma ikimtahadharisha ya kwamba labda mimba aliyobeba Nakhumicha inaweza kuwa huyo ndiye mtoto wa pekee (labda kwa misingi ya jinsia) atakayejaliwa na Mwenyezi Mungu. Hivyo anaweza kujikana na baadaye ikatokea kuwa asipate mtoto wa jinsia hiyo na akaja kutamani tena yule aliyemkana. Ama mtoto atakayezaliwa anaweza kubarikiwa katika miaka ya baadaye akiwa mtu mzima na hivyo Situma atakuwa alipoteza bahati hiyo.

Matumizi ya vipengele teule vya lugha ya Kibukusu mfano msamiati fiche, misemo na methali yanakubaliana na kauli ya Doke (1948) ambaye amezungumzia fasihi na maandishi katika lugha za Kibantu. Anasema lugha ina malighafi yake katika Fasihi Simulizi ya jamii ambayo huathiri matumizi yake. Hivi kwamba ubora wa lugha za Kibantu unatokana na wingi wa msamiati wake ambao unafungamana na Fasihi Simulizi ya jamii husika. Idadi kubwa ya msamiati huu hutumika katika shughuli nyingi za kawaida za wanajamii. Lugha na jamii ni mambo mawili ambayo hayawezi kutenganishwa, kwani lugha ndiyo hufafanua jamii. Kwa hivyo utafiti huu ulibaini matumizi teule ya msamiati wa Fasihi Simulizi ya Babukusu kwa misingi ya uendeshaji wa kesi kitamaduni.

Kutokana na matokeo ya usaili wa Chifu ilibainika kuwa kando na kufahamu tamaduni za jamii, ni kwamba yeye pamoja na naibu wake huwa na uelewa wa sheria za nchi. Aliarifu kwamba mara tu wanapoteuliwa katika nyadhifa hizi za uongozi, serikali hupeleka machifu na manaibu wao kwa mafunzo maalum katika vyuo vya serikali. Hii inawapatia tajriba na maarifa ya kuwawezesha kushughulikia kesi hizi kwa mitazamo yote miwili ya kitamaduni na kisheria. Hivyo wanapoendesha kesi yoyote ile inakuwa rahisi kwao kujumuisha taratibu za utamaduni na Sheria kama zilivyosetiriwa katika katiba ya Kenya iliyoidhinishwa mwaka wa 2010. Chuoni wanapokea mafunzo ya aina mbili ambayo yanahusu yafuatayo:

(i) Mafundisho ya kisheria - iliwapa ujuzi na kuelewa vipengele vya kisheria. Hii huwapa fursa ya kujifahamisha vifungu vya kisheria na ndio sababu wana uwezo wa kuendesha kesi kwa kurejelea mtazamo jumuishi wa kitamaduni na kisheria.

(ii) Mafundisho ya kijeshi - iliwapa ujuzi wa kijeshi kwa ajili ya kujizuia ama kujitetea iwapo watakumbana na hatari.

Vilevile ilibainika kuwa afisi ya Chifu na naibu wake ilikabiliwa na kushughulikia kesi anuwai zinazohusu wanajamii. Kesi hizi zilihusu matatizo kadha ya kijamii kama vile migogoro ya wanandoa, tatizo la mashamba, masuala ya haki za watoto, wizi, ulevi, urithi, ugomvi wa ndugu na madeni. Wakati wa utatuzi wa kesi hizi matumizi ya lugha mara nyingi yaliegemea lugha ya Kibukusu. Hata hivyo kuna wakati ambao kwa sababu kadha kesi ziliendeshwa kwa lugha ya Kiswahili. Hii ni pale ambapo mmoja wa wahusika katika kesi hakuelewi vyema lugha ya Kibukusu. Hali ambayo imetokana na ndoa mseto na uhamaji watu. Maelezo haya yanaafikiana na mhimili wa nadharia ya utafiti unaozungumzia ule ufahamu wa msomaji au msemaji aliye katika nafasi fulani kitamaduni na kihistoria ambayo huchangia kuleta maana ya matini hivyo kufanya mchakato wa kusoma au kuzungumza kuwa hai. Muktadha huu kwa mujibu wa utafiti wetu ulifidiwa na taaluma ya Semantiki ambayo ilitumika kufafanua maana zilizofumbwa kutokana na vipengele vya mwingiliano wa Fasihi Simulizi na taaluma ya Sheria.

Usaili kwa chifu uliendeshwa kwa kurejelea dodoso iliyoandaliwa awali. Maswali yaliyoulizwa yaliibua matokeo mbalimbali ambayo yalisaidia katika kuchanganua data ya utafiti huu. Swali la kwanza lililoulizwa lilitaka kujua mwelekeo unaotumiwa katika uendeshaji wa kesi ili kuwasilisha ujumbe. Chifu, alikubali kuwa kesi zinapoendeshwa mwelekeo mseto ulitumiwa. Mwelekeo ambao ulijumuisha Fasihi Simulizi, tajriba na ujuzi wa taaluma ya Sheria. Taarifa hii ya Chifu ilithibitisha kuwa kuna mwingiliano matini baina ya taaluma hizi mbili hivyo palikuwa na haja ya kufanya utafiti 
ili kubaini ukweli huu. Kurejelea vikao vya tulivyohudhuria ilibainika kuwa kesi zilizotatuliwa katika afisi na mabaraza ziligusia masuala anuwai yanayoathiri wanajamii. Hali hii inakubaliana na maoni ya mtaalam Schenck (2013) ambaye alifanya utafiti kuhusu usomaji wa taaluma ya Sheria kama Fasihi na kwa upande mwingine kuisoma Fasihi kama Sheria. Katika kazi yake ameangalia namna michakato ya kimahakama katika Ufaransa ya kale ilivyoelezea kuhusu Sheria katika muktadha wa kitamaduni. Muktadha ambao unaathiri jamii kwa masuala mbalimbali kama yaliyoelezewa na watafitiwa wa utafiti huu.

Isitoshe, miongoni mwa kesi tulizohudhuria na kuangazia katika mabaraza na mikutano afisini mwa Chifu zilihusu suala la ndoa kama walivyoeleza mtafitiwa. Kwa wa mujibu wa majibu ya watafitiwa hatua ya kwanza katika kukabiliana na kesi ya ndoa ni kubainisha aina ya ndoa husika ili kujua iwapo ni halali au si halali. Uhalali wa ndoa uliathiri mkondo wa kesi. Ndoa halali ni ile ambayo imefuata utaratibu kama inavyohitajika na utamaduni wa jamii ya Babukusu au Sheria ya nchi. Nayo ndoa isiyo halali ni ile ambayo wachumba walichukuana tu na kuanza kuishi bila kufuata utaratibu unaohitajika na jamii. Maelezo ya chifu yalidhihirisha kuwa Sheria ya Kenya inatambua ndoa ya kitamaduni ya Babukusu kuwa halali sawa na ndoa za kitamaduni za jamii zingine katika taifa hili. Kwa misingi hii hivyo basi ni kwamba katika muktadha wa utamaduni wa Babukusu ndoa halali ni ile ambayo mume huwa amefuata taratibu zinazompasa na utamaduni. Hii ndiyo itafanya atambuliwe kama ndiye aliyeoa msichana husika. Mvulana baada ya kumchukua binti wa familia fulani anapaswa kujitambulisha kwa wazazi wa msichana. Hatua ambayo huitwa:

4. Khukhola sisiemiko (kutoa gharama ya msichana kuchukuliwa).

Khukhola ni neno lililo na maana ya kutekeleza na sisiemiko ni kitendo maalum kinachotekelezwa na wasichana kutoka jamii ya binti aliyeolewa katika boma fulani. Baada ya jamii ya kina msichana kutambua alikoolewa binti yao, wasichana kutoka kwao huja asubuhi moja na kuingia katika zizi la ng'ombe kwa kina mvulana na kuchukua ng'ombe aliyewapendeza kisha kuondoka naye bila kuongea na mtu yeyote. Hata wenye boma wakiwaona hawasemi chochote huwaacha tu kuondoka na mnyama huyo bila kulalamika. Hii ilifuatiwa na shughuli ya mvulana kufahamisha jamii ya msichana aliko binti yao. Kujitambulisha huku kulihitaji mvulana kuwatuma watu hasa kina mama kwa wazazi wa msichana kuwajulisha kuwa binti yao yuko mikononi mwake. Aidha, atakuwa anawasihi wasimtafute kwa sababu msichana hajapotea bali yuko katika makao fulani ambapo ni kwake. Ujumbe huu uliandamanishwa na bidhaa hasa nafaka kama vile wimbi, mtama na wimbi. Bidhaa hizi ni kama kitulizo kwa wazazi wa msichana labda kutuliza hasira waliokuwa nayo kutokana na kutoweka kwa binti yao. Kwa hivyo kama utaratibu huu ulifuatwa basi kwa misingi ya utamaduni wa Babukusu basi ndoa hiyo huwa ni halali, inatambuliwa na wazazi hata ukoo. Matokeo haya yanaafikiana na maoni ya Fish (1990) ambaye akirejelea Fasihi Simulizi na Sheria ya Wagiriki anasema taswira tunayopata kutokana na utamaduni wa jamii hii inahusu mikutano ya hadhara, maamuzi ya kesi, malipo mbalimbali na utatuzi wa migogoro. Katika kauli hii tunaona mwingiliano wa utamaduni na Sheria miongoni mwa Wagiriki. Hali ambayo inaingiliana na utafiti huu kwamba kesi za kitamaduni miongoni mwa Babukusu zinaendeshwa katika mikutano ya hadhara ambapo maamuzi kuhusu kesi hizo hutolewa. Vilevile, migogoro ikiwemo ya ndoa inahusisha malipo mbali mbali.

Kutokana na mojawapo ya kikao cha kesi ilionekana kuwa asasi ya ndoa iliibua migogoro mingi na hivyo kusababisha kesi nyingi ilisukuma watafiti kutaka kutambua mambo zaidi yanayohusiana na kesi hizi. Hali hii vilevile ilichochewa na sababu kuwa kati ya kesi zilizohudhuriwa kiasi kikubwa kiligusa suala la ndoa. Hivyo watafiti walifuatilia ili kubaini michakato zaidi inayotumiwa katika kukabiliana na suala hili. Jawabu likawa kuwa afisi ya chifu ilidadisi juu ya mambo kadhaa kuhusu kesi ya ndoa inayoshughulikiwa. Kwanza walitaka kujua iwapo katika ndoa hiyo kulikuwa na watoto ama la. Msemo uliotumiwa ulikuwa ni:

5. Khuba ne lulwibulo (kuwa na uzao ama watoto)

Neno khuba lina maana ya kuwa, nalo ne linamaanisha na ilhali lulwibulo ni kizazi au uzao. 
Msemo una maana ya kutaka kujua kama kuna watoto katika uhusiano huo, idadi yao pia ijulikane kuwa ni wangapi. Watoto ni sehemu muhimu sana katika ndoa ya Kibukusu kiasi kwamba kama wanandoa hawana uwezo wa kupata watoto basi mikakati huwekwa ya kuwapata. Mfano kama mume ndiye ana tatizo la uzazi, mkewe aliruhusiwa na utamaduni kupata watoto na shemeji zake lakini wakaitwa kwa jina la mumewe. Iwapo ni mke basi jamii ilimruhusu mume kuoa mke mwingine ili azae watoto. Kwa hivyo mwenyekiti wa kikao cha kesi baina ya Kizito na Nekesa alitaka kujua iwapo wachumba hawa wana watoto au la. Ilikuwa ni kwa ajili ya kutaka kuwahimiza waishi kwa amani ili wawalee watoto wao vizuri.

Majibu zaidi yaliyotokana na usaili wa Chifu yalibaini kuwa katika jamii hii kiungo kikuu baina ya mke na mume ni watoto. Mara nyingi ndoa ilidumu kwa watoto kuzaliwa baina ya wanandoa. Hata mahari wakati mwingine ililipwa kwa ajili ya watoto. Pia mtoto alilipiwa mahari iwapo alizaliwa nje ya ndoa na mamake akaolewa kwingineko. Kwa hivyo kabla familia ya mvulana haijamchukua mtoto kutoka kwa wazazi wa msichana ni lazima gharama ya kumlea mtoto huyo itolewe. Kesi zinapotatuliwa inakuwa muhimu chifu kujua kama mgogoro husika unawahusu watoto au la. Matumizi ya lugha ya utatuzi kesi hutegemea sababu za mzozo. Kuna misemo na methali ambayo inahusu hali na mambo tofauti tofauti kulingana na suala linaloshughulikiwa.

Kuongezea kwa hayo Chifu na kamati yake ambayo ilijumuisha wazee wa vijiji waliuliza iwapo mahari ilikuwa imelipwa au la. Ikitokea kwamba mahari haijalipwa mume alihitajika kueleza ni kwa nini hajatimiza wajibu huu. Kando na watoto, mahari pia yalichangia pakubwa kuhakikisha kuwa wachumba wanaishi pamoja na kudumisha ndoa yao. Waaidha, ilionyesha kuwa mume anatilia maanani uhusiano wake na mkewe. Mume ambaye hajalipa mahari alichukuliwa kama mwenye mizaha mingi na hayuko tayari kwa maisha ya ndoa. Pia kulipa mahari ilikuwa njia bora ya kushukuru wazazi wa msichana kwa kumzalia mke na kuonyesha kuwa amekubalika katika jamii mpya. Ikiwa kwamba mahari yamelipwa inakuwa rahisi kusuluhisha tatizo katika ndoa husika. Vilevile ilielezwa ikiwa mahari yamelipwa yote au nusu. Stakabadhi za makubaliano baina ya jamii zote mbili huwasilishwa kama idhibati ya mkataba huo.

Hali kadhalika mwenyekiti wa baraza la wazee alisaidia kutoa habari kuwa ndoa ni sehemu pana ya maisha ya wanajamii ya Babukusu kwa sababu ndio inayowezesha ukuaji wa jamii yoyote ile. Jamii hii inaamini kuwa ukuaji wa jamii yao ni kupitia kwa ndoa imara ambazo zinadumisha maadili. Hivyo hali zozote zilizoathiri asasi hii zilivutia mwingilio wa jamii pana ili kuhakikisha inadumu. Ndio sababu baadhi ya kesi zilizowasilishwa zilijumuisha sio tu mwenyekiti bali pia wanajamii kutoka pande zote mbili za msichana na mvulana. Istilahi zaidi zilizotumika katika kuendesha kesi za asasi hii kama vile:

\section{Khucha khutekha (kuenda kupika).}

Khucha ina maana ya kuenda na khutekha ni kupika. Hivyo msemo huu una maana ya kuenda kupika, wanaoenda kupika ni wanawake; msemo mwingine unahusiana na ndoa ni:

\section{Khucha lilia (kuenda kula).}

Lilia ina maana ya kula pia inaweza kumaanisha kuliwa kutegemea na muktadha. Kwa hivyo ina maana ya kuenda kula au kuliwa. Huu pia unarejelea jinsia ya kike kwani wao ndio huenda kwa kina mume bila shaka kula au kuliwa. Pamoja na hiyo msamiati mwingine uliorejelea asasi hii ni:

8. Khukhwalikha (kuenda kushiriki tendo la ndoa).

Msamiati ambao ulimsawiri mwanamke kama aliyeolewa kwa ajili ya kushiriki mapenzi. Hii inatokana na maana yake kiuamilifu. Maana ambayo ilielekea kuwa na hisia za kibabedume kwani ilionyesha kuwa mwanamke katika ndoa jukumu lake ni kukidhi uchu na mahitaji ya mwanamume. Hali hii ilionekana kukosa tasfida na ni ya kumdhalilisha mwanamke katika ndoa. Hata hivyo msamiati huu miongoni mwa watu wa kale ulikuwa wa kawaida na hawakuona fedheha kulitaja. Kufifia kwa matumizi ya msamiati huu pia kumechangiwa na mabadiliko ya kijamii ambayo ni pamoja na ndoa za mseto, ujio wa dini, uhamaji wa watu kuenda mijini, elimu ya vitabu na harakati za wanajamii kuunga mkono usawa wa jinsia. 
Afisi ya Chifu na baraza la wazee baada ya kuthibitisha kuwa taratibu zote za ndoa zilitimizwa, walitumia mikakati iliyohakikisha kuwa wanandoa hao wanapatanishwa. Katika mazingira na utamaduni wa Babukusu utengano au kuachana kwa wanandoa hauruhusiwi. Kesi za ndoa zinaposhughulikiwa kuna kujumuisha ukoo, wazee kutoka pande mbili. Ndoa katika jamii hii ni suala linalihusisha jamii nzima wala si la mtu mmoja ama wazazi wa wanandoa pekee. Kinachoashiria hili ni kwamba mfano mahari inapotolewa haisalii tu katika boma na familia ya akina msichana pekee bali hugawanywa kwa watu wengine wa ukoo. Vilevile wakati wa utoaji wa mahari ya kupeleka kwa kina msichana jamii ya mvulana ilichangiana ili kufikisha idadi ya ng'ombe wanaohitajika na mahitaji mengine yaliyotakikana. Hivyo tatizo linapozuka inakuwa ni shughuli ya kikoa kwa nia ya kuhakikisha amani na umoja unadumishwa.

Ufumbaji wa maana katika uendeshaji wa kesi kuhusu ndoa ulitokea katika miktadha mbalimbali kulingana na aina ya kesi iliyokuwa ikiendeshwa. Katika moja ya kesi hizo tuliyohudhuria mnamo tarehe 09/03/2019 katika afisi ya Chifu kulikuwa na matumizi ya msemo:

\section{Khukhola eng'anana (kufanya mazungumzo ya ulipaji mahari).}

Hapa neno khukhola lina maana ya kufanya, eng 'anana ni hatua ya mwanzo ya kujadili mahari. $\mathrm{Ni}$ shughuli inayohusu wazee kutoka pande mbili za mume na mke, ambao walizungumzia utaratibu na makubaliano ya ulipaji wa mahari. Shughuli hii inaweza kuendeshwa kwa kina mume au kwa kina mke kulingana na walivyokubaliana wazee.

Hali kadhalika katika tukio hilo kuhusu mume ambaye amekosa kuwajibika katika kuishughulikia familia yake kimahitaji, alihimizwa kutekeleza wajibu wake na mshiriki Mama I kwa kuambiwa msemo huu:

10. Niwe papa we lukoba (wewe ndiwe baba wa boma).

Kwa hivyo alipaswa kuhakikisha kuwa familia yake inapata chakula, watoto wana mahala pa kulala, wanaenda shule na mengine yampasayo kufanya kama baba. Hali hii inaweza kuhusishwa na kipengele cha Sheria kinachohusu haki za watoto. Alijulishwa kwamba watoto wana haki kikatiba ya kupata chakula, malazi, mavazi, elimu na usalama. Mambo haya yote wanahakikishiwa na wazazi hasa sana baba ambaye ndiye kichwa cha familia katika jamii hii na kama ilivyo katika jamii nyingi za Kiafrika. Pia alistahili kumtunza mkewe kwa mahitaji ya kimsingi na kutekeleza wajibu wake kama mume. Tukio hili linaenda sambamba na utafiti wa Rutherford, and Rutherford (1992), ambaye kwa kufafanua maoni yake anatoa maelezo ya kesi moja kule Ugiriki. Alitumia maandiko katika kitabu cha 18 cha The Lliad; anaeleza juu ya jamii fulani iliyokuwa ikitafuta haki. Ambapo kikundi cha wanaume kilikusanyika katika ukumbi wa umma ili kujadili kuhusu gharama ya damu ya jamaa wao ambaye alikuwa ameuawa kwa jina Achilles.

Tukio hili la mchakato wa uendeshaji kesi kisheria kuhusu Achilles ni mojawapo ya majadiliano ya wazi kuhusu sheria ya kitamaduni katika jamii ambayo iliishi nyakati zilizotawaliwa na Fasihi Simulizi. Wakati ambao majaji hawakuwa wataalamu waliopata mafundisho yoyote maalum ya uendeshaji wa kesi. Majaji wakati huo waliteuliwa kutoka miongoni mwa wazee ambao walioaminika kuwa na hekima ya kuelewa matukio na tamaduni za jamii kisha kutoa maamuzi kuhusu suala fulani.

Uchunguzi zaidi ilidhihirisha kuwa mzozo wa kinyumbani vilevile ulihusu mume au mke ambaye ameshindwa kutimiza jukumu lake la ndoa. Hili liligusa uhusiano wao kindoa katika chumba cha kulala. Kesi hii ilihusisha vipengele vya Fasihi Simulizi kama vile methali na misemo. Hii nayo vilevile imewakilishwa katika mojawapo ya kesi ambapo mhusika Nekesa alitumia msemo ufuatao kitasfida kuonyesha jinsi mumewe hampi haki yake ya kindoa:

\section{Kamakhuwa ke ebulili (mambo yanayohusiana na malazi).}

Tafsiri ya maneno katika msemo huu ni; kamakhuwa ni mambo, ke ni yanayohusiana na, halafu ebulili ni malazi. Msemo huu kwa jumla uligusia jukumu la mama ama baba kama mchumba wa mwenzake. Ulitumika pale ambapo mmoja wa wachumba alidai haki yake ya tendo la ndoa. Misemo zaidi iliyotumiwa kuhusiana na suala hili ili kupunguza makali ya suala husika ni kama vile: 
12. Khwamenya bakhasi fweng'ene (hapa tunaishi wanawake pekee).

Maneno yanayojenga istilahi hii yana maana zifuatazo khwamenya ni tunaishi, bakhasi ina maana ya wanawake na fweng'ene ni peke yetu. Huu ulihusu mke akidai haki yake ya ndoa kutoka kwa mumewe alisema hivyo wakati anajieleza mbele ya wazee. Nekesa mke wa Kizito anatumia msemo huu ambao tafsiri yake moja kwa moja katika Kiswahili ni kuwa wanaishi kama wanawake tu. Hii ilieleweka kumaanisha mume wake hawajibiki katika tendo la ndoa. Kwa upande mwingine mumewe naye anajitetea kwa kudai haki akisema:

\section{Omukhasi yuno sekanjalikhilao busuma ta} (mke huyu hanipikii sima).

Maneno yake yana tafsiri ifuatayo omukahsi ni mke, yuno ni huyu, sekanjalikhilao ina maana ya hanibandikii mekoni, busuma ni sima na ta ni kukana. Msemo huu uliashiria kuwa mume huyo haonani kimwili na mkewe kwa sababu mke hukataa. Hali hii ililazimu kuwa watafute kiini cha tatizo hilo ili mwongozo mwafaka utolewe. Mambo yalipozidi na kuonekana kuwa suluhisho halipatikani, basi mwenyekiti wa ukoo alihusishwa na ikiwezekana wanapaswa kutoka pande zote mbili ya mume na mke ili kupata muafaka wa tatizo husika.

Kilichobainika katika kesi ya Nekesa na Kizito ni kwamba mume alikuwa na uhusiano wa kimapenzi na mwanamke mwingine nje ya ndoa. Nekesa mke wa Kizito anatoa madai kuwa mumewe anatembea na msichana mmoja kwa jina Judy ambaye anaishi katika nyumba ya kupanga pale sokoni. Hivyo mkewe anatumia msemo:

14. Akendanga ne Judy (yeye hutembea na Judy).

Akendanga hapa ina yeye hutembea, nalo neno ne ni na, kisha $J u d y$ ni jina la msichana anayedaiwa kuwa mpenzi wa mume wake. Kwa hivyo hii ilidhihirisha kuwa mchumba anayeshukiwa anashiriki uzinzi na ndio sababu akifika nyumbani hana hamu na mwenzake. Lugha hii ni fiche kwa kuwa kutembea tu na mtu hakuwezi kuwa tatizo, lakini katika muktadha husika inaeleweka kuwa ni kumuendea kinyume mchumba wako katika ndoa.
Maelezo haya yanakaribiana na maoni ya Fortune (1962) ambaye anasema kuwa kando na muundo wa lugha kimsamiati na kimofolojia, kuna vipengele vingine zaidi vinavyowasilisha ujumbe. Vipengele anavyorejelea kwa mujibu wa utafiti huu ni tanzu za Fasihi Simulizi. Aidha maudhui yanaweza kuwasilishwa kupitia ishara ambazo hugusa hisia za watu. Mfumo huu ulitumika na wasemaji wazuri ambao pamoja na ufundi wao wa lugha, walihusisha ishara na uteuzi mwafaka wa maneno ili kuafikia lengo lao. Miondoko hii huenda sawa na hisia za msemaji. Mtaalam huyu ameangazia lugha teule ya wanenaji jambo ambalo linaendana na utafiti huu.

Pamoja na hayo ni kwamba katika ndoa kunaweza kuwa na dhuluma. Kutokana na ya kikao tulichohudhuria cha kesi na usaili ulioendeshwa kwa mwenyekiti wa kikao hicho ilidhihirika kuwa hali hii ilitajwa kwa msemo ufuatao:

15. Bukulumi bwe mwilia (dhuluma katika ndoa).

Bukulumi lina maana ya dhuluma, bwe ni katika na mwilia nalo ni ndoa. Ilibainika kuwa Nekesa alikuwa anapitia uhasama katika ndoa kutoka kwa mumewe ambaye alimpiga. Ilionekana mumewe alimpiga wakati mwingine bila sababu maalum. Katika jamii ya awali tendo hili lilikuwa jambo la kawaida kwa sababu mume aliaminiwa kutamalaki mke au wake zake kwa kichapo. Pia iliaminika kuwa mwanamke ni muhali kupewa kichapo kabla hajakaa sana katika ndoa ili asije akamea pembe. Msemo uliorejelea hali hii ni:

\section{Khutumia liika (kuvushwa meko).}

Ndio sababu Mzee I anamshauri Kizito kuwa mke akishafanyiwa hivyo inatosha, mazoea ya kumpigapiga mke si jambo zuri. Tafsiri ya msemo huo ni khutumia lina maana ya kuvushwa na liika ni jiko. Kwa hivyo una maana ya mume kumvukisha mkewe meko. Tendo hili lilikuwa la kumkaribisha kikamilifu mke katika ndoa ili asije akavimba kichwa baadaye. Mwanamke ambaye hakupigwa ilichukuliwa kuwa angekuwa na kiburi. Hata hivyo mabadiliko ya kijamii na sheria yameleta mtazamo mpya katika dhuluma za aina yote. Mabadiliko haya yameathiri jamii ya Babukusu pakubwa. Kwa hivyo kuhusu hali kama hii afisi ya chifu hujumuisha kipengele cha sheria 
kinachohusu dhuluma za aina yoyote ile ikiwemo za ndoa. Tahadhari ilitolewa kwa yule anayedhulumu mwenzake kwa misingi na mahitaji ya sheria. Mwelekeo huu unakubaliana na maoni ya White (1985) ambaye anasema kuwa baada ya kuunda mikakati ya usomaji wa sheria taaluma hii ilianza kuhusishwa pakubwa na dhana ya mwingiliano taaluma. Anaona sheria kuwa ni sehemu ya usemaji. Kipengele ambacho pia kinaathiri taaluma ya Fasihi Simulizi. Anakanusha kauli kuwa Sheria na Fasihi Simulizi ni nyanja tofauti, kwa kudai kwamba mwanasheria ni mwanasayansi kwa sababu yeye hajihusishi na data bali huzama katika matini. Usomaji wa Sheria unahitaji kupitia na kudurusu makala, majarida na vitabu mbalimbali. Kisha White (2014) anazidi kusema mwanasheria ni mfasiri anayejishughulisha na lugha ili kutoa tafsiri za hadithi kutoka hadhira moja hadi nyingine.

Kando na hayo wakati mwingine dhuluma za ndoa huweza kusababisha kifo. Kama Mzee I anayerejelea kifo cha mke wa muulana wa Chapili ambacho kilisababishwa na mvulana huyo. Kitendo hiki kikatwika jamii hiyo mzigo wa kulipa fidia ya aliyeaga kwa jamii yake. Kilichobainika ni kuwa katika harakati za kumpiga mkewe akidhani kuwa anamwadhibu, mume aliweza kujipata amesababisha kifo ama kwa kukusudia au la. Hali hii nayo vilevile ililazimu mume husika kutoa malipo kwa jamii ya mke kabla ya kuruhusiwa kumzika. Malipo yaliyotolewa kwa kosa hili yaliitwa:

\section{Khurunga kumurwe (kulipa kichwa).}

Tafsiri ya maneno katika usemi huu ni kama ifuatavyo khurunga ni kulipa na kumurwe ni kichwa. Maneno haya yakichukuliwa kwa maana yake ya kijuujuu huenda yakaeleweka visivyo. Msemo huu unapotumiwa una maana kuwa mtu ameuawa na mwingine, anaweza kuwa ni mke au mume na kifo cha aina hii kinapaswa kufidiwa. Mzee I anatumia mfano huu kumtahadharisha Kizito dhidi ya kuwa na mazoea mabaya ya kumpigapiga mkewe. Tabia hii inaweza ikamletea balaa sawa na mvulana wa Chapili. Alikumbushwa kuwa gharama ya kulipia mtu aliyeuawa ilikuwa juu zaidi. Ilihitaji jamii za pande zote mbili kukaa na kukubaliana ili kupata muafaka kwa namna ambayo itaondoa uhasama licha ya malipo kutolewa. Hivi kwamba hata kama malipo yatatolewa uhusiano mwema uendelee kuwepo. Ndio sababu kila mbinu na njia ilitumiwa ili kupanguza makali ya kosa ambayo pia yalipunguza namna ya hukumu iliyotolewa kwa aliyeua. Msemo mwingine ulioandamana na hali hii ulikuwa khuchukha sifuki; ambapo khuchukha lina maana ya kumwaga nalo sifuki ni damu hivyo kwa ujumla wake linamaanisha kumwaga damu. Kwa lugha ya kueleweka tutasema kuwa ni kuua bila kukusudia.

Ni muhimu kuripoti kuwa mauaji yanaweza kutokea katika miktadha mingine kando na ndoa. Kwa hali yoyote ile kesi inayohusu mauaji ilishughulikiwa kwa njia sawa ili kupata suluhu linalodumisha usalama bali sio uhasama. Gharama ya kutoa malipo ilijadiliwa na kupitishwa na wazee kutoka pande zote mbili. Malipo haya yalitolewa kabla ya mwili wa mwendazake kuzikwa. Kama ilivyo na malipo mengine ya aina ya hii wanyama waliotolewa si wa kufugwa. Wao huchinjwa papo hapo kama kafara ya kufidia kifo cha aliyeuawa na kuondoa laana. Damu ya wanyama hawa iliaminika kutakasa kosa lililotokea kwa pande zote kushiriki mlo pamoja.

Swali kuhusu kwa nini katika mazungumzo wakati wa usuluhishaji wa kesi ulionekana kutumia vipengele kadha vya Fasihi Simulizi kuwasilisha ujumbe. Watafitiwa walijibu kuwa matumizi ya istilahi hizo yalisaidia kupunguza makali na kuficha uwazi. Vilevile ufumbaji wa maana ni kwa ajili ya kuwafanya watoto wasielewe yanayoendelea kwa sababu yangewaharibu. $\mathrm{Ni}$ kwamba wakati mwingine si vyema kuweka mambo wazi ila pana haja ya kutumia tasfida. Hali hii ilioana na namna wanasheria wanavyofumba maana katika lugha ya kisheria. Hivyo ndivyo ilivyo na ufumbaji katika Fasihi Simulizi kupitia tanzu zake kama vile methali, misemo, mashairi na hadithi. Kwa mujibu wa mzee wa baraza ni kwamba mara nyingi katika kesi zilizohusu masuala ya kitamaduni, korti zilitumia uamuzi wa wazee wa ukoo. Hii ilitokana na usaili ulioendeshwa kwake nyumbani tarehe 10/04/2019.

Kutokana na mahojiano hayo aidha ilibainika kuwa kuna kesi ambazo zilipelekwa kortini lakini zikarudishwa katika afisi ya chifu ama kamati ya wazee. Kesi hizi zilishughulikiwa na kamati maalum ya kusuluhisha migogoro, maamuzi ya 
kamati hii yalipelekwa kortini ili kutathminiwa. Hali kadhalika mzee huyo alirejelea mahakama ya kitamaduni ya Sirisia ambayo awali ilishughulikia kesi miongoni mwa Babukusu kitamaduni. Alisema kuwa wakati huo hali ilikuwa afadhali kwa sababu wanajamii ya Babukusu walielekeza kesi zao huko moja kwa moja. Kesi katika afisi ya Chifu zinashughulikiwa na kamati za kutatua migogoro ambazo zina wanachama wafuatao:

- Chifu ndiye mwenyekiti.

- Manaibu chifu - wanachama.

- Wananchi - wanachama (hawa ni watu wenye tajriba ya maisha, waadilifu na wenye ufahamu wa utamaduni ambao huteuliwa kutoka jamii).

Katika kiwango cha afisi ya naibu wa Chifu kamati ya kutatua migogoro ilikuwa na wanachama wafuatao:

- Naibu wa Chifu ndiye mwenyekiti

- Wazee wa vijiji katika kata ndogo anayoongoza.

- Wanachama wa nyumba kumi.

Mtaalamu wa sheria katika kamati hizi ni Chifu au naibu wake kutegemea ni kiwango gani kesi inaendeshwa. Mwenyekiti ambaye ni Chifu au naibu wake ndiye ana jukumu la kutoa mwelekeo kwa kuzingatia taaluma zote mbili Fasihi Simulizi na Sheria. Hii ni kwa sababu mafundisho wanayopokea kabla ya kuanza kazi yalijumuisha vipengele vya Sheria. Kutokana na hoja kuwa wao ni sehemu ya jamii husika hivyo wana ujuzi katika utamaduni wa Babukusu. Vilevile wanachama wengine ni watu wenye tajriba pana ya maisha, utamaduni na mahusiano ya jamii ya Babukusu.

Kando na masuala ya ndoa utafiti ulibaini kuwa mzozo wa mashamba pia ulikuwa tatizo sugu miongoni mwa jamii hii. Kesi za mashamba zilijitokeza kwa namna mbili; ya kwanza kuna kesi zilizohusu shamba la ukoo na namna ya pili ilihusu shamba ambalo mzazi amenunua kwa pesa zake. Mzozo wa shamba aidha unaweza kuwa juu ya mpaka unaohusisha majirani au hata watu wa ukoo mmoja na mambo mengine mengi. Mfano wa kesi ya shamba tuliohudhuria ilihusu makanyako, msamiati huu ulikuwa na maana ya kuingia katika shamba la mtu mwingine bila idhini yake. Kesi hii ilikuwa tarehe 03/10/2019 katika afisi ya naibu wa
Chifu. Shamba la ukoo ni lile ambalo mtu alirithi kutoka kwa wazazi wake. Babu humuachia baba, naye baba hupaswa kuwapokeza watoto wake na vivyo hivyo kwa vizazi vitakavyofuata. Mzozo wa aina ya pili unaohusu shamba alilonunua mzazi, uamuzi wa jinsi ya kugawa hutegemea mnunuzi mwenyewe ambaye ni mzazi. Akipenda agawe watoto wake ama akose kuwagawa au hata auze. Hapa familia haina usemi, mzazi ambaye ndiye mnunuzi ana mamlaka yote juu ya shamba hilo ya jinsi atakavyolitumia.

Tukirejelea mfano wa kesi aina hii kulidhihirika matumizi anuwai ya lugha ya Kibukusu kimafumbo. Mifano yake ni kama ifuatavyo:

18. Khubilika chiswa (kutengeneza mahali pa kukusanya na kuchotea kumbikumbi).

Neno khubilika lina maana ya kutengeneza mahali (palipoonyesha dalili za kutoka kwa kumbikumbi) kutumia vijiti kwa kuvikunja kisha kufunika kwa blanketi ili kujitayarisha kuzoa wadudu hao. Nalo chiswa ni kumbikumbi. Wadudu hawa wanatoka mara nyingi mwezi wa nane na tisa na wanapendwa sana na wanajamii hii. Wakati mwingine hutumika kama kitoweo. Kwa hivyo Khubilika chiswa ni kuandaa mahali maalum hasa kwenye kichuguu ambapo pameonyesha dalili ya kutoa kumbikumbi ili uweze kuwazoa kwa urahisi.

Kesi hiyo ya Makanyako ilichukuwa muda na hivyo kudhihirisha matumizi mengi ya vipengele vya Fasihi Simulizi. Metrine anatumia methali ifuatayo kueleza jinsi alivyomfahamu shemeji yake kuwa na hasira.

19. Owakibaya nie wakimanya (aliyeifuga ndiye anayeijua).

Maana ya maneno katika methali hii ni kama ifuatavyo; Owakibaya ni yule ambaye amefuga, nie inamaanisha yeye ndiye na wakimanya inasimamia anayeijua. Kwa hivyo methali hii ina maana ya kwamba mtu ambaye amefuga mnyama fulani ndiye anayefahamu tabia zake. Unapoishi na mtu unajua hulka zake na kile anachoweza kufanya. Metrine alimjua shemeji yake Elijah kama mtu mwenye hasira hivyo aliogopa kumwendea wazungumze kuhusu kuharibu mimea yake shambani. Mwelekeo huu unakubaliana na utafiti wa msomi Wanjala (2015) ambaye alishughulikia 
mwingilianotanzu unaojitokeza wakati wa utendaji wa mivigha. Matokeo ya utafiti wake yalibainisha nafasi muhimu ya Fasihi Simulizi katika kuhifadhi mila na desturi za jamii husika. Sawa na matokeo ya uchunguzi uliofanywa juu ya utafiti huu yalitoa taswira kamili ya mwingilanotaaluma uliopo baina ya Fasihi Simulizi na Sheria. Pia yalionyesha namna taaluma hizi mbili zinaweza kushirikiana na kuingilana ili kutekeleza wajibu wake katika jamii ya Babukusu.

Maoni zaidi kuhusu mzozo wa shamba la ukoo kutoka kwa mtafitiwa wetu yalionyesha kuwa afisi ya chifu hufanya kazi kwa karibu sana na ukoo. Ilionekana kuwa kesi zinapoendeshwa kitamaduni ukoo huwa ni kiungo muhimu kwa sababu viongozi wa ukoo wanaaminika kuwa na ufahamu kuhusu watu wa ukoo wao. Aidha kila ukoo huwa na kamati ndogo ndogo zinazoshughulikia mambo tofauti tofauti ya kijamii. Miongoni mwa kamati hizi ni ile inayojishughulisha na mashamba. Kesi za aina hii hata mahakama ilionelea kwamba zisuluhishwe na Chifu akiungana na ukoo wa wahusika. Maelezo haya yaliafiki matamshi ya Jaji mkuu wa mahakama nchini mheshimiwa David Maraga tarehe 29/01/2018 kupitia idhaa ya redio ya Jambo. Jaji Maraga alielekeza kwa kutoa ushauri kuwa kuna baadhi ya kesi ambazo hazistahili kufikishwa kortini bali zinaweza kusuluhishiwa nyumbani kupitia afisi ya Chifu kwa ushirikiano na ukoo. Alitaja kesi kama vile kutoelewana kuhusu mipaka ya mashamba na zile za urithi. Kwamba zisuluhishwe vijijini na watu wanaofahamu wanaozozana, kule bila shaka watakoawajibika ni chifu, kamati yake na viongozi ukoo wa wanajamii husika.

Mashauri yake yalituelekeza kwenye kesi moja ya Chifu akitoa maelekezo katika baraza, kijiji cha Khamoto kuhusu mizozo ya mashamba. Data ilikusanya kutumia mbinu ya uchunzaji-shiriki ambapo watafiti waliweza kuhudhuria na kushuhudia katika muktadha asilia kesi zikiendeshwa kitamaduni.

Katika kesi na mikutano aina hii maamuzi ya ukoo ndio yalitoa mwongozo katika kesi za mashamba, kazi ya Chifu ni kutoa mwelekeo kwa kujumuisha utamaduni na sheria. Mwelekeo anaotoa Chifu haupaswi kuathiri maamuzi ya ukoo. Maoni ya Chifu kwa upande mwingine yalitegemea utaratibu uliofuatwa na ukoo. Jambo la kimsingi ni kwamba utatuzi wa migogoro ya mashamba ni jukumu la kamati ya ukoo inayohusu mashamba. Jambo lingine kuhusu mzozo wa shamba la ukoo ni kwamba watoto wana uwezo wa kupinga ili mzazi asiuze au kulitumia kiholela. Haya vilevile yanaoana na sheria inayohusu umiliki wa mashamba, kwamba mzazi hana uhuru wa kuuza shamba liwe la ukoo ama alilonunua bila ya kukubaliana na mkewe na watoto. Kuonyesha kwamba walikubaliana na hatua hiyo wanapaswa kutia sahihi stakabadhi za uuzaji wa shamba. Iwapo mnunuzi hatazingatia haya na kesi iwasilishwe mahakamani huenda akapoteza kipande hicho cha ardhi. Mtafiti alitambua kuwa katika ufumbaji wa maana vipengele kadha vya Fasihi Simulizi ya Babukusu vilitumika wakati wa uendeshaji kesi kitamaduni zilizohusu mashamba.

Utafiti ulitambua misemo zaidi wakati wa kushughulikia tatizo hilo la shamba kama ifuatayo; kulikuwa na msemo:

20. Khukhwekesia omwana nio alima (kuonyesha mtoto pahali pa kulima).

Neno khukhwekesia lina maana ya kuonyesha, omwana ni mtoto, nio ina maana ya pahali pa na alima ni kulima. Kwa hivyo ni kuonyesha mtoto wa kiume mahali anapostahili kulima mara nyingi inatakikana awe yule ambaye ameoa. Kwa kufanya hivyo shamba huwa halijagawiwa kwani ni kumuonyesha mahali pa kulima tu ili kukimu familia yake kwa chakula wala asiendelee kutegemea wazai. Kisha baadaye kuna:

21. Khukhwekesia omwana nio kombakha (kuonyesha mtoto mahali atajenga nyumba).

Sawa na 20, Khukhwekesia ni kuonyesha, omwana ni mtoto hapa likirejelea wa jinsia ya kiume, nio ni mahali pa na khuombakha ni kujenga. Ilitarajiwa kwamba mvulana baada ya kuoa alipaswa kutoka katika simba (nyumba inayojengewa wavulana ili wasilale nyumba moja na wazazi) na kujenga nyumba yake. Kwa hivyo mzazi alimuoyesha ni wapi alistahili kujenga. Hata hivyo kuna wakati licha kwamba mvulana hajaoa bado alihitajika kutoka mradi awe amefika umri wa kujitegemea. Katika hatua hii pia huwa shamba bado halijagawishwa. Kuonyeshwa mahali pa kujenga inaweza kuwa kujenga esimba hii ni nyumba ya 
wanamolala wavulana kabla ya kuoa, au mvulana anaweza kuwa anaonyeshwa mahali pa kujenga nyumba ya kuishi na mkewe.

Shamba linakuja kugawiwa baadaye. Shughuli ya kugawa shamba miongoni mwa Babukusu ni khukhwara lulwakha. Khukhwara ni kupasua ilihali lulwakha ni mpaka msemo sawa na huo ni khuana likongwe. Khuana lina maana ya kupeana na likongwe ni mmea wa mkonge. Mmea huu ndio ulitumiwa kuweka mpaka baina ya shamba la watu wawili. Hiyo ndiyo hatua ya mwisho ya urithishaji wa shamba kutoka kwa mzazi kuendea watoto wake. Iwapo kulitokea mgogoro kuhusu ugawaji wa shamba ulishughulikiwa na ukoo chini ya mwenyekiti ambaye amepewa mamlaka na ukoo. Mahakama vilevile husita kuangazia kesi za aina hii hasa iwapo baba mzazi angali hai kwa sababu ana uhuru wa kupeana kulingana na anavyopenda. Ugawaji shamba miongoni mwa Babukusu ni jukumu la baba kwa wakati mwingine anaweza kushirikisha ukoo. Ukoo ulihusishwa katika migogoro ya mashamba kwa sababu koo nyingi zina kamati za kushughulikia mashamba na migogoro mingineyo.

Aidha kulikuwa na kesi ambazo zilihusu madeni katika jamii. Watafiti walipata fursa ya kuhudhuria kikao cha kesi ya aina hii kwenye afisi ya chifu mnamo tarehe 25/4/2019 na kurekodi yaliyojiri. Katika mfano huu wa kesi kuna matumizi ya hapa na pale ya vipengele vya Fasihi Simulizi. Kwanza kulitumika msemo:

22. Khukhalaka khale kamakhuwa (kuja kama tayari mmefanya maamuzi).

Neno khukhalaka lina maana ya kuamua, nalo khale lina maana ya tayari kisha kamakhuwa ni mambo. Msemo hivyo basi ulimaanisha kuwa wao wamekuja kwa Chifu kama tayari wameamua kesi. Istilahi hii ilitumiwa na Chifu kuwashauri wahusika waache kesi ichukue mkondo wake kulingana na vile watakavyojieleza na kutoa ushahidi. Vilevile kuna matumizi ya msamiati mumbarasia kwa maana ya kutokea kandokando na kutaka kuchangia kwa jambo bila kupewa ruhusa. Aidha kulitumika neno khunonosiamo ambao ni msamiati wa kejeli ulioonyesha ukosefu wa heshima katika mazungumzo yako. Kwamba mmoja wao alichangia bila ruhusa ya Chifu na aliyosema hayakuhusiana na suala linaloshughulikiwa. Hali kadhalika Chifu alitumia methali miongoni mwazo ikiwa ni:

23. Nandakambilwa kakona khumwanda kwa enjoli (mtu ambaye hafuati ushauri hujipata katika shida kubwa).

Msamiati uliotumika kujenga methali hii una tafsiri zifuatazo nandakambilwa ni mtu ambaye haambiliki wala hasemezeki. Mtu ambaye hazingatii mawaidha au ushauri, daima anatenda mambo visivyo na akishauriwa habadiliki. Kakona ina maana kulala kwa, khumwanda ni barabara kubwa na pana, kwa ni kwenye nayo enjoli ni fisi. $\mathrm{Ni}$ methali ambayo maana yake ya juu ni mtu ambaye hatilii maanani mashauri anayopewa hujipata kalala kwenye barabara wanayopitia fisi. Maana ya ndani ni kuwa mtu asiyesikia mawaidha mara nyingi hujipata ameingia kwenye shida kubwa zaidi. Hivyo ilikuwa inawahimiza wahusika katika kesi hiyo kusuluhishia mambo yao hapo ili kuepuka gharama na uhasama unaoweza kutokea kufuatana na maamuzi ya korti. Namna kesi hii ilivyoendeshwa inakubaliana na mawazo ya Dworkin (2015) ambaye anasema mahitaji ya sheria ya jamii fulani hutegemea mwelekeo wao katika kutambua uhalali wa taratibu zilizopo. Iwapo wanajamii wana mwelekeo sawa, anauliza watawezaje kushiriki katika migogoro inayohitaji utatuzi wa kisheria? Mielekeo inayotofautiana ndiyo huzua mitafaruku miongoni mwa wanajamii. Dworkin (k.h.j.) anadokeza kwamba nadharia yoyote ya sheria inapaswa kuwa na tafsiri hai inayolenga kutatua migogoro hai inayowakabili wanajamii. Tafsiri ambayo inaafiki hali halisi ya kile kinachofasiriwa na kukionyesha kama kinachotimiza lengo lake.

Kwa mujibu wa matokeo katika sehemu hii kuhusu lengo la kwanza la utafiti ilibainika kuwa nadharia ya utafiti ya mwingilianomatini ilikuwa mwafaka. Hii ni kutokana na mhimili wa nne unaodai kuwa mwingilianomatini ni namna ya kufasiri matini. Kwamba ni hali ambapo matini hukopa maneno na dhana kutoka matini nyingine na kuitumia katika kufafanua maana katika matini iliyokopa. Hivyo matini za Sheria hukopa kutoka matini za Fasihi Simulizi ili kufafanua dhana mbalimbali kwa wahusika katika kesi. Kila mwandishi kabla ya kuandika matini yake na anapokuwa katika mchakato wa kuandika, yeye husoma matini 
iliyoandikwa kabla ya matini yake na kuirejelea. Kwa hivyo yeye hukopa kutoka matini au diskosi tangulizi na zilizopo. Hivyo basi kazi ya mwandishi yeyote huwa na mwangwi wa matini nyingine ambazo huwa amezirejelea moja kwa moja au kwa njia isiyo ya moja kwa moja na kuihusisha katika kazi yake. Tukirejelea muktadha wa utafiti huu ni kwamba kesi zinapoendeshwa kitamaduni zina mwangwi wa Sheria ambazo pia kwa wakati mwingine hurejelewa moja kwa moja. Lugha ya kisheria ni tata sawa na lugha teule ya kifasihi simulizi.

Vilevile kupitia kwa usaili uliofanyiwa mzee wa jamii ya Babukusu ambaye ni mmoja wa watendaji wa mivigha ya lufu utafiti uliweza kupata data inayohusiana na lengo la kwanza. Watendaji hawa huitwa baswali kumuse lakini sherehe yenyewe ni khuswala kumuse. Khuswala ni kukanyaga na kumuse ni mahali maalum ambapo jamii ya marehemu imeteua pafanyiwe sherehe hii. Msemo huu una maana kuwa yule mtendaji huongea huku akitembea katika sehemu maalum na jamii imeketi kumsikiza. Lufu ni sehemu ya sherehe ya mazishi ambayo huja siku tatu baada ya maziko ya mwendazake. Hata hivyo utendaji huu ni kwa marehemu ambaye ni wa jinsia ya kiume. Kwa hivyo sherehe ilifanyiwa wanaume pekee tena wa umri mkubwa bali si wa makamo kurudi chini. Kando na usaili huu watafiti walipata kuhudhuria utendaji wa mivigha hii na kukusanya data kutokana na matukio yaliyoafiki malengo ya utafiti.

Kutokana na muktadha wa matumizi ya lugha katika mivigha hii kuna vipengele kadha vya Fasihi Simulizi vinavyodhihirika. Mwanzo hadithi yenyewe ni fumbo kwa maana ilivyoendelezwa na kusimuliwa ni kuwatahadharisha watoto wa marehemu na uzao wake dhidi ya kiburi, dharau, hali ya kutojali na kuchochea wengine. Tahadhari ni kwamba mambo hayo yanaweza kumletea mtu madhara. Siku moja utakuja kupata aliyekuzidi kwa yote hayo na kutumia mtego akakunasa kisha ukajiingiza kwenye shida. Kwenye hadithi kuna matumizi ya vipengele kadha vya Fasihi Simulizi vinavyojitokeza ikiwa ni pamoja na msemo kakefwe kamakhale; kakefwe ni mambo haya ni yetu na kamakhale ina maana ya tangu zamani. Ni msemo unaorejelea kifo kama tukio ambalo limekuwepo tangu jadi. Kwa hivyo ulitumika kuhimiza waliofiwa kujipa moyo kwa kuwa kifo si jambo geni, kimekuwa na mwanadamu tangu zama za mababu zetu. Vilevile kuna matumizi ya methali mojawapo ikiwa ni:

\section{Omukesi sekebeka ta (mwerevu hajinyoi).}

Msamiati wake una maana zifuatazo omukesi ni mtu mwerevu, khwibeka ni kujinyoa na ta ni hawezi. Maana ya methali hii kuwa hata mtu awe mwerevu kiasi gani hawezi kujinyoa. Inapofika hapo lazima atafute kinyozi ili atekeleze wajibu huo. Tunaweza kuihusisha na ile methali ya Kiswahili inayosema kinyozi hajinyoi.

Aidha katika mwendelezo wa utendaji huo wa lufu kuna matumizi ya misamiati migumu kama vile lirhambo kwa maana ya mtu mkuu ambaye alikuwa anategemewa katika familia; sikili kwa maana ya kiburi; sisianyi kwa maana ya shamba lilo katika msitu na endalo ni sehemu ndogo katika shamba ambapo zilipandwa mboga. Mwendelezo huu wa utendaji unaoana na maoni ya White (1985) ambaye anahoji kuwa hadithi ni sawa na Sheria kwa kuwa inaturuhusu kuhisi, kuwa na tajriba hata kuwazia hali ambazo hazituhusu binafsi. Ni namna ya kuibua hisia ili kukabiliana na hali fulani mahsusi ya maisha ya binadamu. Anafafanua sheria kuwa ni seti ya matini inayopaswa kusomwa kwa kulenga hali mbalimbali zinazomhusu binadamu kutumia njia mbalimbali. Kwake hadithi inaweza kutumiwa kutoa hukumu katika Sheria au kuamua kesi.

Matokeo haya yalikubaliana na mhimili wa pili wa nadharia ya utafiti wetu ambao unaeleza kuwa matini zinaingiliana na kuchangiana, hivyo kuamini kuwa matini moja inajitegemea ni kupotoka. Pamoja na hayo mwingilianomatini ulihusisha pia mitindo fulani ya kiusemi na misimbo ambayo imesaidia katika uashiriaji wa kazi za baadaye. Hivyo mwingilianomatini sio urejeleaji tu wa kazi fulani katika kazi nyingine, bali pia ni ule mwingiliano wa mitindo ya kiuashiriaji. Lugha ya fasihi kwa kiasi kikubwa huwa ni ya kiishara kwa sababu maana huwa imefumbwa katika semi zinazotumiwa, sawa na lugha ya kisheria. Ndiposa tunaona katika baadhi ya utendaji wa mivigha ya utamaduni wa Babukusu kuna mwingiliano wa vipengele vya Fasihi Simulizi. Kahini alitumia hadithi, wimbo, misemo na methali. Hali kadhalika uendeshaji wa kesi 
kitamaduni katika afisi ya Chifu ulijumuisha vipengele vya Sheria.

Hivyo basi ni muhali kusema kuwa matini za kifasihi simulizi huwa zinachota, kunukuu, kugeuza, kuiga kwa namna ya kufumba, kubeza au kurejelea kwa njia moja au nyingine matini za kisheria. Hali kadhalika tanzu zenyewe za Fasihi huingiliana na kuchangiana kama anavyosema Tracey (1948), anazungumzia kipera cha nyimbo katika Fasihi Simulizi. Anasema kuwa kipengele hiki hutumika pamoja na tanzu nyingine kama vile ngano, mivigha na sherehe mbalimbali. Anaendelea kusema kuwa nyimbo hujitokeza katika nathari ikiwa ni kwenye hadithi, masimulizi, vitendawili na methali. Utukiaji wa nyimbo au uimbaji huwa ni kwa ajili ya kutofautisha nathari na ushairi. Kwamba kuna umuhimu wa nyimbo katika Fasihi Simulizi ikiwemo kuvunja kaida na kuleta burudani. Mwingiliano wa nyimbo na tanzu nyingine huathiri Fasihi Simulizi ya jamii nyingi. Hii pia ilichangia kuimarisha utendaji wa tanzu nyingine za Fasihi Simulizi. Watafitiwa waliohojiwa, makala ya Fasihi Simulizi ya Babukusu na makala ya sheria yaliyorejelewa yalionyesha mawazo halisi ya wanajamii ya Babukusu katika kukabiliana na kesi mbalimbali kitamaduni na kisasa.

\section{HITIMISHO NA MAPENDEKEZO}

Kwa mujibu wa matokeo ya utafiti imeonekana kwamba mwingiliano taaluma unaodhihirika kati ya Fasihi Simulizi na Sheria unaiwezesha jamii ya Babukusu kukabiliana na hali zinazoikumba. Ni wazi kuwa wakati wa uendeshaji kesi za kitamaduni vipera vya fasihi simulizi ya Babukusu kama vile jazanda, methali, misemo, tashbihi, maghani, mivigha, nyimbo na ngano zilitumika ili kukuza uhusiano bora na maelewano miongoni mwa wanajamii. Katika kutekeleza wajibu huu maana ya vipera hivi huwa imefumbwa kwa lengo la kupunguza makali. Tulipendekeza kuwa: Utafiti mwingine unaweza kufanywa ili kubainisha mwingiliano taaluma uliopo baina ya Fasihi Simulizi na taaluma za historia, dini, isimu na sayansi ili kuonyesha uwezekano wa kuingiliana kwa taaluma hizi katika kushughulikia mahitaji ya jamii.

\section{MAREJELEO}

Dorsch, T. S. (1965). Classical Literary Criticism Aristotle: On the Art of Poetry; Horace: On the Art of Poetry; Longinus: On the Sublime.

Bassey, M. (1999). Case study research in educational settings. McGraw-Hill Education (UK).

Creswell, J. W., \& Creswell, J. D. (2017). Research design: Qualitative, quantitative, and mixed methods approaches. Sage publications.

Enon, J. C. (1998). Educational research, statistics and measurement. Kampala: Makerere University.

Doke, C. M. (1948). The basis of Bantu literature. Africa: Journal of the International African Institute, 18(4), 284-301.

Dworkin, R. (2015). How law is like literature. In Law and Literature (pp. 45-62). Routledge.

Fish, S. (1990). Almost Pragmatism: Richard Posner's Jurisprudence.

Fortune, J. (1962). Melon of Ecstasy. London: Hutchinson. ISBN 978-1853754708

Gay, R. (2000). Educational Research Conference for Analysis and Application. (Toleo la 4). New Jersey.

Rutherford, R. B., \& Rutherford, R. B. (Eds.). (1992). Homer: Odyssey Books xix and xx. Cambridge University Press.

Jilala, H. (2014). Athari za kiutamaduni katika tafsiri mifano kutoka matini za Kitalii katika makumbusho za Tanzania (Doctoral dissertation, University of Dar es Salaam).

Kombo, D. K., \& Tromp, D. L. (2006). Proposal and thesis writing: An introduction. Nairobi: Paulines Publications Africa, 5, 814-30.

Kothari, C.R. (2004). Research Methodology: Methods and Techniques (Toleo la 2): New Delhi: New Age International (P) Ltd. 
Kothari, C. R. (2013). Research Methodology: Methods and Techniques (Toleo la 3): New Delhi: New Age International (P) Ltd.

Kress, G. (2008). Meaning and learning in a world of instability and multiplicity. Studies in Philosophy and Education, 27(4), 253-266.

Leedy, P. D. (1997). Practical Research: Planning and Design. (Toleo 6). New Jersey: PrenticeHall, Inc.

Msokile, M. (1992). Misingi ya Hadithi Fupi. Dar es Salaam: Dar es Salaam Printing

Press.

Oso, Y. W. \& Onen, D. (2005). A general Guide to Writing Research Proposal and Report. Toleo la 2. Kampala: Makerere University Printery.

Robson, C. (2002). Real-World Research: A Resource for Social Scientists and Practitioner - Researchers (2 ${ }^{\text {nd }}$ ed.). Oxford: Blackwell Publishers Ltd.

Schenck, H. (2000). The influence of negative advertising frames on political cynicism and politician accountability. Human Communication Research. Thousand Oaks: GA Publication

Spata, G. (2006). The Statesman Yearbook: Politics, Cultures and Economics of the World. Palgrave: Macmillan Ltd

Tracey, M. (1948). The Decline and Fall of Public Service Broadcasting and Production of Political Television. Colorado: University of Colorado Boulder Press.

Wanjala, S. F. (2015). Mwingilianotamzu katika Fasihi Simulizi ya Kiafrika: Mfano wa Embalu na Mwaka Kogwa. Tasnifu ya Uzamifu, Chuo Kikuu cha Kenyatta.

White, J. B. (1985). Law as Rhetoric, Rhetoric as Law: The Arts of Cultural and Communal Life. University of Chicago Law Review.

White, J. B. (2014). What Can a Lawyer Learn from Literature? Harvard Law Review: Harvard University Press. 